\title{
Study Protocol of the International Spinal Cord Injury (InSCl) Community Survey
}

\author{
Mirja H. Gross-Hemmi, PhD, Marcel W. M. Post, PhD, Cristina Ehrmann, PhD, Christine Fekete, PhD, \\ Nazirah Hasnan, PhD, MRehabMed, MBBS, James W. Middleton, MD, PhD, Jan D. Reinhardt, PhD, \\ Vegard Strøm, PhD, and Gerold Stucki, MD, MS, \\ on behalf of the International Spinal Cord Injury Community Survey (InSCI) Group
}

\begin{abstract}
Objective: The Learning Health System for Spinal Cord Injury (LHS-SCI) is an initiative embedded in the World Health Organization's (WHO's) Global Disability Plan and requires the statistical collection of data on the lived experience of persons with SCI to consequently formulate recommendations and policies. The International Spinal Cord Injury (InSCI) community survey has been developed as an initial step to gain information about the lived experience of persons with SCI within and across diverse nations.

Design: InSCI is a multinational community survey based on the International Classification of Functioning, Disability and Health Core Sets for SCI and involves 28 countries from all six WHO regions. The study will be implemented in 2017. Overall aims, guiding principles on sampling strategies, data collection modes, and reminder management are described.

Conclusions: InSCI will be the first survey to be conducted simultaneously in many countries and in all six WHO world regions that identifies the factors associated with functioning, health, and well-being of persons living with SCI. Expected results of the survey will be used for the basis of conducting stakeholder dialogs for policy reforms designed to improve the functioning, health maintenance, and well-being of persons with SCI.
\end{abstract}

Key Words: Spinal Cord Injuries, Community Survey, International Classification of Functioning, Disability, Health

(Am J Phys Med Rehabil 2017;96(Suppl):S23-S34)

$S_{\mathrm{cos}}^{\mathrm{p}}$ pinal cord injury (SCI) is a relatively low-prevalence, highcost health condition that affects between 250,000 and 500 000 persons worldwide each year. An SCI has far-reaching consequences for a person's functioning and health, as affected persons experience impairments of sensory and motor function below the lesion level. ${ }^{1}$ SCI and associated health problems lead to premature mortality and increased risk for lower physical and mental health compared to the general population. ${ }^{1,2}$ In interaction with environmental factors and personal resources, an SCI may lead to lower participation in social and economic activities. ${ }^{3,4}$ As a result, the lived experience

From the Swiss Paraplegic Research, Guido A. Zäch Institute, Nottwil, Switzerland (MHG-H, MWMP, CE, CF, JDR, GS); University of Groningen, University Medical Center Groningen, Department of Rehabilitation Medicine, Center for Rehabilitation, Groningen, the Netherlands (MWMP); Brain Center Rudolf Magnus and Center of Excellence in Rehabilitation Medicine, University Medical Center Utrecht and De Hoogstraat, Utrecht, the Netherlands (MWMP); Department of Rehabilitation Medicine, University of Malaya, Kuala Lumpur, Malaysia (NH); John Walsh Centre for Rehabilitation Research, Kolling Institute, Northern Sydney Local Health District and Sydney Medical School Northern, University of Sydney, Sydney, Australia (JWM); Institute for Disaster Relief and Reconstruction, Sichuan University and Hongkong Polytechnic University, Sichuan, Chengdu, China (JDR); Department of Health Sciences and Health Policy, University of Lucerne, Lucerne, Switzerland (JDR, GS); Sunnaas Rehabilitation Hospital, Nesoddtangen, Norway (VS); and ICF Research Branch (a cooperation partner within the WHO Collaborating Centre for the Family of International Classifications at the German Institute of Medical Documentation and Information (DIMDI)), Germany (GS).

All correspondence and requests for reprints should be addressed to: Mirja H. GrossHemmi, PhD, Swiss Paraplegic Research, Guido A. Zäch Institute, Nottwil, Switzerland.

The authors declare that they do not receive any financial benefits for this study. This manuscript or abstract has not been presented in any form elsewhere.

Financial disclosure statements have been obtained, and no conflicts of interest have been reported by the authors or by any individuals in control of the content of this article.

Copyright (C) 2017 Wolters Kluwer Health, Inc. All rights reserved.

ISSN: 0894-9115

DOI: 10.1097/PHM.0000000000000647 of persons with SCI has increasingly become the focus of research, going beyond the description of one's health state into domains of functioning and disability, as characterized by the WHO's International Classification of Functioning, Disability and Health (ICF) ${ }^{5}$ and summarized in the International Perspectives on SCI (IPSCI) report. ${ }^{1}$ Weaknesses in health and rehabilitation systems can worsen the burden of disability and lead to poorer health outcomes; reduced well-being; and increased risk of poverty, dependence, and restricted participation. ${ }^{5}$

Yet, owing to inconsistent or inadequate data sources, comparable figures on the incidence and functional consequences of SCI, as well as health services and social provisions for persons with SCI, are largely unavailable for most countries. ${ }^{1}$ As recommended by the IPSCI report, systematic data collection and evaluation of the lived experience of SCI, as well as the state of SCI-relevant health and rehabilitation systems, provide essential information on what society can do to improve the situation of persons with SCI and enhance their health, functioning, and well-being. ${ }^{1}$

The Learning Health System for Spinal Cord Injury (LHS-SCI) ${ }^{6}$ is an initiative rooted in the WHO's Global Disability Action Plan 2014-2021 Better health for all people with disability and in particular with its main objectives of reducing barriers and improving access to health services, assistive technology, and community-based rehabilitation as well as strengthening relevant and internationally comparable data collection. ${ }^{7}$ The LHS-SCI achieves its overall goal of continuously improving the lived experience of persons with SCI in three ways: (i) generating data by means of an international SCI (InSCI) community survey and description of the societal response to SCI; (ii) implementing recommendations 
by means of national stakeholder dialogs; and (iii) building SCI-relevant policy and research capacity.

A comprehensive description of the lived experience of SCI in different cultural settings is urgently needed as stated by the IPSCI report ${ }^{1}$ and constitutes the starting point for an international learning experience among diverging health systems within LHS-SCI. InSCI will be implemented in 28 countries (as of February 2016) in 2017 and potentially be repeated at 5-year intervals to obtain longitudinal and contemporary information of the lived experience of persons with SCI across these nations. The participating countries are from all of the six WHO regions (Fig. 1) and represent different stages of economic development and health care systems, allowing for comparative analysis. The first community survey of the Swiss Spinal Cord Injury (SwiSCI) cohort study served as model for the development of the InSCI study protocol. ${ }^{8-11}$ As with SwiSCI, InSCI will use the ICF, ${ }^{12}$ which provides a framework for data generation by conceptualizing functioning, disability, and health as a dynamic interaction between an individual's health condition and contextual factors. ${ }^{12,13}$ The study aim of InSCI is to comprehensively describe the lived experience of persons with SCI on an international level. More specifically, InSCI tries to describe and identify the determinants of levels of functioning, health, and well-being of persons living with SCI within and across countries. The guiding hypotheses for the analysis of the InSCI survey are the following:

- Functioning, health, and well-being vary between countries and WHO regions.

- Variation in functioning, health, and well-being is related to variation in self-reported environmental factors.

- Variation in functioning, health, and well-being is related to variation in self-reported personal factors.

- Variation in functioning, health, and well-being between countries and WHO regions is partly attributable to macrolevel differences in health systems and policies and general economic characteristics.

In this paper, we report on the overall methodology of the first InSCI survey, including the recruitment rationale, the
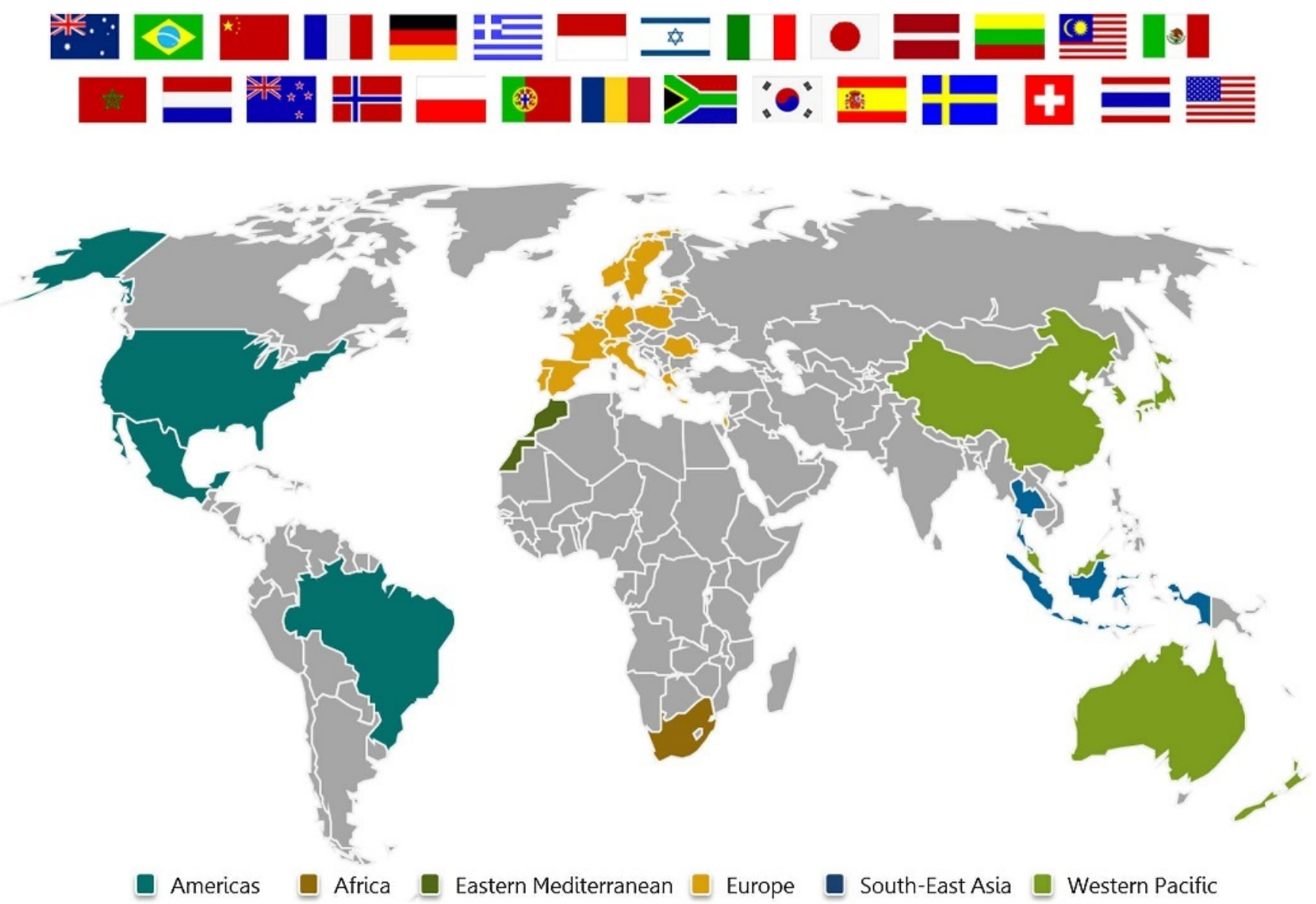

The InSCI community survey includes the following countries: Australia, Brazil, China, France, Germany, Greece, Indonesia, Israel, Italy, Japan, Latvia, Lithuania, Malaysia, Mexico, Morocco, The Netherlands, New Zealand, Norway, Poland, Portugal, Romania, South Africa, South Korea, Spain, Sweden, Switzerland, Thailand, USA.

FIGURE 1. Participating countries of the $\mathrm{InSCl}$ community survey. 
logistic aspects, and the general analysis plan, recognizing that in some countries, the operational implementation may vary according to local conditions.

\section{METHODS}

\section{Design}

The InSCI community survey is a cross-sectional multinational survey including 28 countries from all six WHO regions and is scheduled for 2017, with the potential to be repeated at 5-year intervals. Researchers from various countries were invited for the study during conferences of the supporting societies: International Society of Physical and Rehabilitation Medicine (ISPRM) and International Spinal Cord Society (ISCoS); and all who volunteered to do so participate in the study. Data will be collected using a self-report questionnaire with mixed-mode data collection options (e.g., paper-pencil, telephone interviews, online). National study centers are responsible for the conduct of the survey in their respective country. Besides the development of country-specific sampling and data collection strategies, each national study center develops a strategy for reminding nonresponders. Swiss Paraplegic Research in Nottwil, Switzerland, acts as coordinating institute providing guidance and support to the national study centers with regard to sample design, data collection, storage and archiving, and analysis. Details of each country's national study protocol and optional additional modules are briefly described elsewhere in this issue (see individual country reports).

\section{Target Population and Sampling Frame}

Eligible persons are adults 18 years or older having sustained a traumatic SCI (including cauda equina syndrome) or nontraumatic SCI. Selection of participants adheres to a two-step process: First, potential participants will be identified based on selected codes from the International Classification of Diseases (ICD-10). Selected codes for SCI are in accordance with two studies ${ }^{14,15}$ that showed that these ICD-10 codes are the most commonly used and reliable codes associated with SCI (Table 1). In a second step, available medical information of selected patients will be reviewed, and upon confirmation of an SCI, patients will be included in the study. Eligible subjects are residents of the respective country who live in the community with SCI, are able to respond in one of the available language translations of the questionnaire, and have provided informed consent. Excluded are persons with spinal cord damage due to congenital etiologies such as spina bifida, or neurodegenerative disorders such as multiple sclerosis, amyotrophic lateral sclerosis (ALS), or peripheral nerve damage such as Guillain-Barré Syndrome. Persons who are inpatients receiving first rehabilitation or first acute care at the time of the study are also excluded owing to lack of community experience with SCI.

Owing to unavoidable differences between countries in existence and access to contact databases for individuals living with SCI in the community, InSCI allows for multiple sampling frames for the recruitment of participants. Each country defines its sampling design process. To ensure high scientific
TABLE 1. Selection criteria for $\mathrm{SCl}$

\begin{tabular}{|c|c|}
\hline ICD-10 Code & Description \\
\hline G82 & Paraplegia and tetraplegia \\
\hline G82.0 & Flaccid paraplegia \\
\hline G82.1 & Spastic paraplegia \\
\hline G82.2 & Paraplegia, unspecified \\
\hline G82.3 & Flaccid tetraplegia \\
\hline G82.4 & Spastic tetraplegia \\
\hline G82.5 & Tetraplegia, unspecified \\
\hline G83.4 & Cauda equina syndrome \\
\hline $\mathrm{S} 12$ & Fracture of the neck \\
\hline $\mathrm{S} 12.0$ & Fracture of first cervical vertebra \\
\hline $\mathrm{S} 12.2$ & Fracture of other specified cervical vertebra \\
\hline S13.0 & Traumatic rupture of cervical intervertebral disk \\
\hline $\mathrm{S} 13.2$ & Dislocation of other and unspecified parts of neck \\
\hline S13.4 & Sprain and strain of cervical spine \\
\hline $\mathrm{S} 14$ & Injury of nerves and spinal cord at neck level \\
\hline $\mathrm{S} 14.0$ & Concussion and edema of cervical spinal cord \\
\hline $\mathrm{S} 14.1$ & Other and unspecified injuries of cervical spinal cord \\
\hline S17 & Crushing injury of neck \\
\hline $\mathrm{S} 19$ & Other and unspecified injuries of neck \\
\hline $\mathrm{S} 22$ & Fracture of rib(s), sternum and thoracic spine \\
\hline $\mathrm{S} 22.0$ & Fracture of thoracic vertebra \\
\hline $\mathrm{S} 23.1$ & Dislocation of thoracic vertebra \\
\hline $\mathrm{S} 24$ & Injury of nerves and spinal cord at thorax level \\
\hline $\mathrm{S} 24.0$ & Concussion and edema of thoracic spinal cord \\
\hline $\mathrm{S} 24.1$ & Other and unspecified injuries of thoracic spinal cord \\
\hline $\mathrm{S} 28$ & $\begin{array}{l}\text { Crushing injury of thorax and traumatic amputation } \\
\text { of part of thorax }\end{array}$ \\
\hline $\mathrm{S} 29$ & Other and unspecified injuries of thorax \\
\hline $\mathrm{S} 32$ & Fracture of lumbar spine and pelvis \\
\hline $\mathrm{S} 32.0$ & Fracture of lumbar vertebra \\
\hline S33.1 & Dislocation of lumbar vertebra \\
\hline S34.0 & Concussion and edema of lumbar spinal cord \\
\hline $\mathrm{S} 34.1$ & Other injury of lumbar spinal cord \\
\hline $\mathrm{S} 34.3$ & Injury of cauda equina \\
\hline $\mathrm{S} 38$ & $\begin{array}{l}\text { Crushing injury and traumatic amputation of part of } \\
\text { abdomen, lower back and pelvis }\end{array}$ \\
\hline $\mathrm{S} 39$ & $\begin{array}{l}\text { Other and unspecified injuries of abdomen, } \\
\text { lower back and pelvis }\end{array}$ \\
\hline $\mathrm{T} 02.0$ & Fractures involving head with neck \\
\hline $\mathrm{T} 02.1$ & Fractures involving thorax with lower back and pelvis \\
\hline T04.1 & $\begin{array}{l}\text { Crushing injuries involving thorax with abdomen, } \\
\text { lower back and pelvis }\end{array}$ \\
\hline $\mathrm{T} 04.2$ & $\begin{array}{l}\text { Crushing injuries involving multiple regions of } \\
\text { upper } \operatorname{limb}(\mathrm{s})\end{array}$ \\
\hline T06.0 & $\begin{array}{l}\text { Injuries of brain and cranial nerves with injuries of } \\
\text { nerves and spinal cord at neck level }\end{array}$ \\
\hline T06.1 & $\begin{array}{l}\text { Injuries of nerves and spinal cord involving other } \\
\text { multiple body regions }\end{array}$ \\
\hline T09.3 & Injury of spinal cord, level unspecified \\
\hline T09.4 & $\begin{array}{l}\text { Injury of unspecified nerve, spinal nerve root and } \\
\text { plexus of trunk }\end{array}$ \\
\hline T91.1 & Sequelae of fracture of spine \\
\hline T91.3 & Sequelae of injury of spinal cord \\
\hline
\end{tabular}


quality standards, the study center will evaluate the sampling design process of each collaborating country to optimize procedures and harmonize data collection if needed. After approval of the process, national standard operational procedures are developed to attain high standardization of processes and ultimately high scientific quality. To counteract potential selection bias, a hierarchy of defined sampling frames is proposed as follows: national or regional registry of persons with SCI, databases of academic or level I trauma hospitals, databases from specialized rehabilitation centers, databases from disabled people's organizations or insurance agencies, samples from previous cohort studies or a combination of the aforementioned databases. Countries are advised to refrain from opportunistic recruitment strategies with unidentified sampling frames (e.g., open access internet links; social media; snowball sampling), since these methods prevent an evaluation of sampling bias and correction for nonresponse. In addition, countries are advised to collect all eligible persons and, after a check of their survival status, draw a random sample of the desired size from this group based on the estimated minimal sample size (see next paragraph). Details on the recruitment in the various countries, if different, are described in the individual country reports in this issue.

Based on a power analysis using data from the SwiSCI community survey, ${ }^{8}$ a minimal target sample size of 200 participants per country is expected to provide sufficient power for comparative analysis of functioning outcomes across countries. Expecting a response rate of $50 \%$, the sampling frame by country should at least contain 400 individuals. ${ }^{8}$ The power analysis used person ability scores in five ICF domains (b1 mental functions, b2-b8 functions of body systems, d4 mobility, d5 self-care, and d6-d9 involvement in life situations), which were calculated using Rasch analysis. ${ }^{11}$ To estimate minimal sample size, a $10 \%$ difference on these ability scores between subgroups was defined as the minimal important difference, with a power of $80 \%$ and $\alpha$ error of 0.05 . Since subgroups may not be of equal size, the consequences of variations in the relative size of two comparative samples were considered for sample size

TABLE 2. Sample size estimates

\begin{tabular}{|c|c|c|c|c|c|c|}
\hline \multirow[b]{2}{*}{ ICF-based Scale } & \multirow{2}{*}{$\begin{array}{l}\text { Standard Deviation of } \\
\text { Standardized Scale }\end{array}$} & \multicolumn{5}{|c|}{$\begin{array}{c}\text { Ratio of Two } \\
\text { Subgroup Samples }\end{array}$} \\
\hline & & 1 & 2 & 3 & 4 & 5 \\
\hline b1 Mental functions & 0.177 & 102 & 114 & 136 & 160 & 180 \\
\hline $\begin{array}{l}\text { b2-b8 Functions of } \\
\text { body systems }\end{array}$ & 0.151 & 74 & 84 & 100 & 115 & 132 \\
\hline d4 Mobility & 0.217 & 150 & 168 & 200 & 235 & 270 \\
\hline d5 Self-care & 0.246 & 194 & 219 & 256 & 300 & 348 \\
\hline $\begin{array}{l}\text { d6-d9 Involvement in } \\
\text { life situations }\end{array}$ & 0.194 & 122 & 138 & 164 & 190 & 216 \\
\hline
\end{tabular}

Estimated minimum sample sizes to detect a $10 \%$ difference for five standardized ICF-based scales using two samples (subgroups) of varying relative size (ratio range, 1-5) with power 0.8 and alpha 0.05 for the five ICF-based scales.

Estimates were derived using the power and sample size application in Stata (version 13.1; Stata Corp). The gray area indicates minimal sample size estimations of 200 or less. calculation (Table 2) ${ }^{8,16}$ For subgroups of equal size, sample sizes vary between 74 and 194 and are moderately sensitive to size ratio.

\section{Medical Ethics}

As an international survey, InSCI adheres to universal ethical standards, such as the Declaration of Helsinki ${ }^{17}$ and national regulations governing research involving human subjects. Compliance with national laws and regulatory approvals by institutional review board or ethical committee are mandatory for all countries. Informed consent will be sought from each subject or the subject's legally authorized representative, in accordance with national regulations. Each country will specify whether informed consent will be obtained written or verbally. All data will be de-identified before being uploaded to a common database.

\section{Data Collection Tools}

\section{The InSCI Questionnaire-The Person's Perspective}

The study aim of the InSCI community survey is to comprehensively describe the lived experience of persons with SCI on an international level. The underlying InSCI data model is based on a similar approach as the WHO's World Health Survey, which used key components for health as basis for questionnaire construction. ${ }^{18}$ For the InSCI data model, the ICF framework $^{12}$ acts as guidance in selecting the most appropriate ICF categories to fully describe the lived experience of SCI, that is, to capture what matters most to affected persons. The InSCI data model is based on categories included in the Brief ICF core set for SCI, long-term context, ${ }^{19}$ and ICF rehabilitation $\operatorname{set}^{7}$ complemented by selected psychological-personal factors, ${ }^{20}$ lesion characteristics, and appraisal of health and well-being. This resulted in a total of 47 ICF categories to be covered by the InSCI questionnaire (Table 3 ). The goal was to develop a data model that comprehensively captures the lived experience of persons with SCI but at the same time is practicable in participant burden (i.e., not too lengthy). ${ }^{21,22}$ To date, no single measurement instrument exists that covers all relevant ICF domains to comprehensively measure the lived experience of SCI. Existing and sound measurement instruments often focus on specific domains; and hence, the InSCI source questionnaire had to be compiled from various existing data collection tools and original questions. The argumentative process of question selection to operationalize the data model was guided by the four principles of efficiency, feasibility, comparability, and truth and discrimination. ${ }^{23}$ Entire scales of sound and short data collection tools were used whenever applicable (e.g., Short Form (SF)-36 for the assessment of vitality and mental health). If the use of entire data collection tools was not feasible, for example, because they involved too many questions, single questions from general disability or SCI-specific tools were preferred, which comprised at least cognitively tested questions. The selection of questions for the InSCI questionnaire has been an iterative consensus process of internationally renowned experts in the field of SCI. A detailed description of the data model and the development of the InSCI questionnaire is described in detail elsewhere in this issue. ${ }^{24}$

Briefly, body functions are covered by subscales of the SF-36 (vitality and mental health), ${ }^{25}$ questions from the Brief 
TABLE 3. The InSCI data model and data collection tools

\begin{tabular}{|c|c|c|c|c|c|}
\hline ICF Code & ICF Category & RS & SCI LT & Data Collection Tool & No. Questions \\
\hline \multicolumn{6}{|c|}{ Body Functions } \\
\hline b130 & Energy and drive functions & & & SF-36 Vitality Subscale & 4 \\
\hline b134 & Sleep functions & & & New (SCI-SCS-format) & 1 \\
\hline b152 & Emotional functions & & & SF-36 Mental Health Subscale & 5 \\
\hline b270 & $\begin{array}{l}\text { Sensory functions related to temperature and } \\
\text { other stimuli }\end{array}$ & & & SCI-SCS & 1 \\
\hline b280 & Sensation of pain & & & SCI-SCS (1), BPI (1) & 2 \\
\hline b410-b429 & Functions of the cardiovascular system & & & SCI-SCS & 1 \\
\hline b420 & Blood pressure functions & & & SCI-SCS & 1 \\
\hline b430 & Hematological system functions & & & SCI-SCS & 1 \\
\hline b440 & Respiration function & & & SCI-SCS & 1 \\
\hline b455 & Exercise tolerance functions & & & MDS & 1 \\
\hline b525 & Defecation functions & & & SCI-SCS & 1 \\
\hline b620 & Urination functions & & & SCI-SCS & 2 \\
\hline b640 & Sexual functions & & & SCI-SCS & 1 \\
\hline b710 & Mobility of joint functions & & & SCI-SCS & 1 \\
\hline b730 & Muscle power functions & & & Level and completeness of lesion & 2 \\
\hline b735 & Muscle tone functions & & & SCI-SCS & 1 \\
\hline b810 & Protective functions of the skin & & & SCI-SCS & 1 \\
\hline $\mathrm{b}$ & [General question on body functions] & & & $\begin{array}{l}\text { Open question on additional } \\
\text { health problems }\end{array}$ & 1 \\
\hline \multicolumn{2}{|c|}{ Total questions body functions } & & & & 28 \\
\hline \multicolumn{6}{|c|}{ Body structures: } \\
\hline $\mathbf{s 1 2 0}$ & Spinal cord and related structures & & & Level and completeness of lesion & - \\
\hline $\mathbf{s 4 3 0}$ & Structure of respiratory system & & & SCI-SCS & - \\
\hline s610 & Structure of urinary system & & & SCIM-SR & - \\
\hline $\mathbf{s 8 1 0}$ & Structure of areas of skin & & & SCI-SCS & - \\
\hline \multicolumn{6}{|c|}{ Activities \& Participation } \\
\hline d230 & Carrying out daily routine & & & New (MDS-format) & 1 \\
\hline d240 & Handling stress and other psychological demands & & & MDS & 1 \\
\hline d410 & Changing basic body position & & & SCIM-SR (1), SCI-FI AT (2) & 3 \\
\hline d415 & Maintaining a body position & & & MDS (1), MDS-format (1) & 2 \\
\hline d420 & Transferring oneself & & & SCIM-SR & 1 \\
\hline d445 & Hand and arm use & & & MDS (1), SCI-FI AT (1) & 2 \\
\hline d450 & Walking & & & SCIM-SR & \\
\hline d455 & Moving around & & & SCIM-SR + MDS & 2 \\
\hline d465 & Moving around using equipment & & & SCIM-SR & \\
\hline d470 & Using transportation & & & MDS & 2 \\
\hline d510 & Washing oneself & & & SCIM-SR & 2 \\
\hline d520 & Caring for body parts & & & SCIM-SR & 1 \\
\hline d530 & Toileting & & & SCIM-SR & 3 \\
\hline d540 & Dressing & & & SCIM-SR & 2 \\
\hline d550 & Eating & & & SCIM-SR & 1 \\
\hline d570 & Looking after one's health & & & MDS (1), new (1) & 2 \\
\hline d640 & Doing housework & & & MDS & 1 \\
\hline d660 & Assisting others & & & MDS & 1 \\
\hline d710 & Basic interpersonal interactions & & & New (MDS-format) & 1 \\
\hline d770 & Intimate relationships & & & MDS & 1 \\
\hline d850 & Remunerative employment & & & $\begin{array}{l}\text { ILIAS (6), SwiSCI (1), } \\
\text { MDS (2), ERI (2) }\end{array}$ & 11 \\
\hline $\mathrm{d} 845$ & Acquiring, keeping and terminating a job & & & MDS & 1 \\
\hline d920 & Recreation and leisure & & & MDS & 1 \\
\hline \multicolumn{5}{|c|}{ Total activity and participation } & 42 \\
\hline
\end{tabular}


TABLE 3. (Continued)

\begin{tabular}{|c|c|c|c|c|c|}
\hline ICF Code & ICF Category & RS & SCI LT & Data Collection Tool & No. Questions \\
\hline \multicolumn{6}{|c|}{ Environmental Factors } \\
\hline e110 & Products or substances for personal consumption & & & NEFI-SF & \\
\hline e115 & $\begin{array}{l}\text { Products and technology for personal use in } \\
\text { daily living }\end{array}$ & & & & 1 \\
\hline e120 & $\begin{array}{l}\text { Products and technology for personal indoor and } \\
\text { outdoor mobility and transportation }\end{array}$ & & & NEFI-SF & 2 \\
\hline e125 & Products and technology for communication & & & NEFI-SF & 1 \\
\hline e135 & Products and technology for employment & & & SwiSCI & 1 \\
\hline e150 & $\begin{array}{l}\text { Design, construction and building products and } \\
\text { technology of buildings for public use }\end{array}$ & & & NEFI-SF (1), SwiSCI (1) & 2 \\
\hline e155 & $\begin{array}{l}\text { Design, construction and building products and } \\
\text { technology of buildings for private use }\end{array}$ & & & NEFI-SF & 1 \\
\hline e165 & Assets & & & NEFI-SF & 1 \\
\hline e225 & Climate & & & NEFI-SF & 1 \\
\hline e310 & Immediate family & & & NEFI-SF & 1 \\
\hline e320 & Friends & & & NEFI-SF & 1 \\
\hline e340 & Personal care providers and personal assistants & & & NEFI-SF (1), new (1) & 2 \\
\hline e355 & Health professionals & & & MDS & 2 \\
\hline e425 & $\begin{array}{l}\text { Individual attitudes of acquaintances, peers, } \\
\text { colleagues, neighbors and community members }\end{array}$ & & & NEFI-SF & 1 \\
\hline $\mathrm{e} 460$ & Societal attitudes & & & NEFI-SF & 1 \\
\hline e450 & Individual attitudes of health professionals & & & MDS & 2 \\
\hline e570 & Social security services, systems and policies & & & MDS & 1 \\
\hline e580 & Health services, systems and policies & & & MDS (3), NEFI-SF (1) & 4 \\
\hline $\mathrm{e} 590$ & $\begin{array}{l}\text { Labour and employment services, systems } \\
\text { and policies }\end{array}$ & & & SwiSCI & 1 \\
\hline \multicolumn{5}{|c|}{ Total environmental factors } & 26 \\
\hline \multicolumn{6}{|c|}{ Personal Factors } \\
\hline & Sociodemographic and personal characteristics & & & $\operatorname{MDS}(5)$, new (1) & 6 \\
\hline & $\begin{array}{l}\text { Position in the immediate social and } \\
\text { physical context }\end{array}$ & & & MA SSS (1), MDS (1), new (1) & 3 \\
\hline & Personal history and biography & & & New & 1 \\
\hline & Thoughts and beliefs & & & $\begin{array}{l}\text { MSES (2), GSES (2), MDS (2), } \\
\text { WHOQoL Disability (1) }\end{array}$ & 7 \\
\hline & Motives & & & MDS & 1 \\
\hline & Patterns of experience and behavior & & & GBS & 1 \\
\hline Total persor & tors & & & & 19 \\
\hline \multicolumn{6}{|c|}{ Appraisal of health and well-being } \\
\hline & Subjective well-being & & & WHOQoL-BREF & 6 \\
\hline & General health & & & SF-36 & 2 \\
\hline Total apprai & health and well-being & & & & 8 \\
\hline \multicolumn{6}{|c|}{ Lesion characteristics } \\
\hline & Etiology, time of SCI & & & $\operatorname{ISCoS}(1)$, SwiSCI (1) & 2 \\
\hline Total SCI cl & eristics & & & & 2 \\
\hline Total InSCI & onnaires & & & & 125 \\
\hline
\end{tabular}

ICF categories printed in bold are part of the InSCI data model.

RS, ICF rehabilitation set; SCI LT, Brief ICF Core Set for SCI, long-term context.

Abbreviations for data collection tools: BPI, Brief Pain Inventory; ERI, Effort-Reward Imbalance Questionnaire; GBS, General Belongingness Scale; GSES, General Self-Efficacy Scale; ILIAS, International Labour Market Integration Assessment for Spinal Cord Injury; ISCoS, International Spinal Cord Association, Template for Demographics; MA SSS, McArthur Scale of Subjective Social Status; MDS, Model Disability Survey; MSES, Moorong Self-Efficacy Scale; SCI-FI AT, Spinal Cord Injury—Functional Index-Assistive Technology; NEFI-S, Nottwil Environmental Factors Inventory, Short form; SCIM-SR, Spinal Cord Independence Measure for Self-Report; SCI-SCS, Spinal Cord Injury Secondary Conditions Scale; SwiSCI, Swiss Spinal Cord Injury Cohort Study; WHOQoL-BREF, World Health Organization Quality of Life-BREF. 
Pain Inventory ${ }^{26}$ and adapted questions from the Spinal Cord Injury Secondary Conditions Scale. ${ }^{27}$ Activity and participation are mainly assessed by questions from WHO's Model Disability Survey (MDS) ${ }^{28}$ the Spinal Cord Independence Measure for Self-Report, ${ }^{29}$ and the Spinal Cord Injury Functional Index. ${ }^{30}$ Evaluation of work integration and health care services involve questions taken from different tools. Environmental factors will mainly be assessed by the Nottwil Environmental Factors Inventory Short Form ${ }^{31}$ and questions from the MDS. ${ }^{28}$ Relevant personal factors will be measured using questions from the General Self-Efficacy Scale, ${ }^{32}$ the Moorong Self-Efficacy Scale, ${ }^{33}$ the General Belongingness Scale, ${ }^{34}$ and the MDS. ${ }^{28}$ Additionally, basic sociodemographic and socioeconomic characteristics will be gathered as personal factors. Information on lesion characteristics include selfreported severity and completeness of lesion, etiology, and date of SCI. Appraisal of health and well-being will be assessed with the General Health scale of the SF- $36^{25}$ and a 5-item selection of World Health Organization Quality of Life-BREF, ${ }^{35}$ respectively. In total, the InSCI questionnaire comprises 125 questions, and its estimated time for completion is 30 to 45 minutes.

A multidisciplinary team with expertise in measurement, statistics, survey research, information technology, and documentation developed a paper-pencil version of the InSCI questionnaire that serves as template for all countries. The InSCI questionnaire can be self-administered (paper-pencil or online questionnaires, available for use on desktop, tablet, or smartphone) or interviewer-administered (face-to-face or telephone interview). A first draft of the questionnaire was discussed with the national leaders and coordinators of the participating InSCI countries at the ISCoS (Montréal, 2015) and ISPRM (Berlin, 2015) conferences. Feedback from these conferences has been incorporated, and a final draft of the questionnaire has been discussed with experts in respective fields.

For the various translations of the InSCI questionnaire, cross-cultural adaptation based on the guidelines proposed by Epstein et al. ${ }^{36}$ will be applied. These guidelines are designed to maximize the attainment of semantic, idiomatic, experiential, and conceptual equivalence between the English InSCI reference questionnaire and the translated versions. This guarantees both appropriate linguistic translation and cultural adaptation to maintain consistency and the content validity of the InSCI questionnaire across the different participating countries. As a general translation guideline of the InSCI questionnaire, participating countries are advised to only adapt the questionnaire (e.g., altering content or design components) when substantial improvement for a specific sociocultural context is attained. The order of questions and response categories cannot be changed. Maintaining equivalence of the semantics of a phrase has priority above literal translation. Therefore, if a phrase cannot be properly translated by simply translating the words, the appropriate viable translation may be a translation that includes adaptation of content, format, or other feature. Unless validated translations of data collection tools are available, the instructions, questions, and response options will be translated in a three-step process involving two independently translated questionnaire versions, which will be synthesized, checked, discussed, and approved by an expert committee within the national study group. ${ }^{36}$

In a consecutive pretesting phase, difficulties in the questionnaire and study design have been assessed in each country to ensure comparability and equivalence of data. The pretest of the English reference version of the InSCI questionnaire has been performed in the United States and included focus group interviews to test content validity, and the assessment of test-retest reliability. Pretesting of the translated InSCI questionnaire including persons with SCI in all other countries has been recently completed and additionally helped improve the final version of the InSCI questionnaire. Based on the issues raised during translation and pilot testing, decisions on the final version of the InSCI questionnaire have been discussed by researchers from the study center, the chair of the scientific committee, and two international advisors at the end of August 2016.

\section{Variables of the Health System and Economic Resources-The Societal Perspective}

The InSCI research framework allows both national (within country) and cross-national (between countries) analyses. To make this possible, information on specific variables of the health system and economic resources variables, such as community rehabilitation and return-to-work programs, health and accident insurance, and social welfare systems, will be collected to describe the current stage of economic development and health care systems in each country. These variables are potentially important environmental factors or interest to the outcomes of the InSCI study and can be used as factors in the quantitative analyses of functioning, health, and well-being of persons living with SCI. For a detailed list of these variables, see Pacheco Barzallo and Gross-Hemmi ${ }^{37}$ in this issue.

\section{Data Entry, Management, and Access}

For data entry, password-secured web-based entry forms will be used. To guarantee data security, only study participants and dedicated staff of the respective national study centers will have access. This allows both continued data entry on a case-by-case basis and real-time monitoring of accrual rates. In addition, the study center may advice individual countries on operational issues to ensure maximal subject participation.

Study data will be de-identified, harmonized, and centrally stored on a password-protected database server. The study center will be entrusted with the monitoring and coordination of the data collection process, the combining of national data files, and archiving of the data. For country-specific national modules, the countries may use either the central database or their own local database. Personal data of participants will be stored in each country's local database. Both study data and personal data will contain a unique identifier (InSCI ID number) allowing for the linkage of the data sets when required. In addition to the strict de-identification of study data, 
country-level information will also principally be de-identified in reporting of functioning outcomes.

Access to the central database will be password-protected, monitored, and restricted to registered users. Access to the national study database holding personal data will be restricted to a small group of authorized study personnel under the responsibility of the national study center. All study staff will sign a declaration of adherence to data protection regulations.

Upon closure of the InSCI community survey, all data will be systematically checked to ensure that the subject was eligible and the informed consent was signed, and nonsensical responses will be resolved based on standardized operating procedures. The national data will be available to the research teams of each participating country at any time. All researchers intending to use InSCI survey data will have to submit a formal research proposal to the InSCI scientific committee.

\section{Data Analysis}

\section{Statistical Adjustment for Response Bias, and Data Analysis Plan}

Possible country-specific response bias will be estimated by the study center through a unit nonresponse analysis that looks at targeted participants who did not participate in the survey, based on existing personal participant's characteristics (e.g., sex, age, lesion level, date of onset of SCI). To account for nonresponse bias, inverse probability weights based on the propensity of response will be computed for each country. The study center will also account for item nonresponse (missing item response data). If possible, logical imputations with values unambiguously inferred from other response options will be made. Otherwise, data will be imputed both with single imputation and multiple imputation methods, ${ }^{38,39}$ as the preferred use of single imputation or multiple imputation data may depend on the application and previous experience of the researchers. Rasch analysis is the principal validation tool in InSCI to compute and ensure conceptual coherence and cross-national equivalence of ICF-based scores. ${ }^{11,31}$

\section{Data Analysis Plan for the Lived Experience Across Countries}

The data analysis of the InSCI survey consists of a twofold approach. First, the perceived lived experience of people with SCI (the person's perspective) will be analyzed with regard to items of the InSCI questionnaire, which will then be contrasted to variables of the health system to determine the influence of the countries' health systems on the lived experience (the societal perspective). ${ }^{40}$ To achieve this, the analysis plan will analyze both the person's perspective and the societal perspective separately, as follows:

1) Data analysis plan for the lived experience and participation in the society - the person's perspective

First, functioning profiles across all functioning domains based on the prevalence of functioning problems among the population with SCI will be created. Heat graphs ${ }^{41}$ will be used to visualize the extent of functioning problems in both, SCI samples and SCI subgroups identified as meaningful for comparing information across different countries, namely, SCI type (tetraplegia, paraplegia), etiology (traumatic and nontraumatic), SCI degree (complete/ incomplete), age (16-30 years, 31-45 years, 46-60 years, $61-75$ years, and 76 years or older), and sex (male, female). ${ }^{42}$

Second, the complexity of functioning in relation to SCI will be explored using graphical models. Based on this innovative statistical approach, we will be able to examine the association between aspects of functioning and identify the areas of the lived experience that are most influential for optimizing the health of people with SCI.

A graph is defined as a visualization of $(\mathbf{V}, \varepsilon)$, where $\mathbf{V}$ denotes the vector of the functioning variables of interest (also called the nodes of the graph) and $\varepsilon$ a set of edges representing the dependence between those functioning variables. The edges can be undirected $(x-y)$ or directed $(x \rightarrow y$ or $x \leftarrow y$ ). Undirected graphs will be used to visualize mutual association between functioning variables, whereas directed acyclic graphs will be used to represent the effect of hypothetical interventions in each functioning variable on people's health. The undirected graph, called a skeleton, will be estimated using the PC algorithm implemented by Kalisch et al. ${ }^{43}$ In this algorithm, a series of conditional independence tests (starting with three functioning variable and then increasing the set of functioning variables step by step) are carried out for eliminating the edges from a complete graph, that is, one within which all variables (nodes) are connected. The final edges in the skeleton indicate some strong dependence that cannot be explained by conditioning on other variables.

To identify which aspects of functioning may most importantly contribute to optimizing health, intervention calculus developed by Peter Sprintes and Clark Glymour and implemented in the pcalg $\mathrm{R}$ package will be applied. ${ }^{44}$ Setting in the skeleton all edges connected with the selfreported health variable so that they are directed toward the self-reported general health variable at the end will allow us, by using observational data, to quantify the effect on health that is achieved by setting one functioning variable to a particular value (in this case, the value "no problem because of a hypothesized intervention"). Third, we will investigate the relation between different aspects of functioning and contextual factors. Aspects of functioning and environmental barriers will be aggregated into clinically meaningful domains of functioning (e.g., mobility, self-care, domestic life) by maintaining conceptual coherency with the ICF and ensuring robust psychometric properties of the metric obtained following the approach developed for the SwiSCI community survey. ${ }^{45}$ The Rasch measurement model will be applied to derive interval scales for the identified subdomains. ${ }^{46}$ For existing associations between body functions and activities and participation subdomains, the effect of environmental barriers will be studied by comparing moderator hypothesis, mediator hypothesis, and direct effect hypothesis. In addition, we will adjust for variation in age (in years), time since injury, sex, SCI type (paraplegic/tetraplegic), etiology (traumatic/nontraumatic), SCI degree (complete/incomplete), 
and education. Personal factors evaluated with various measurement instruments will also be considered once their Rasch scores have been estimated. ${ }^{47}$

2) Data analysis plan for the influence of the health system on the person's lived experience - the societal perspective

Owing to the hierarchical structure of the expected data, a multilevel approach is inevitable to determine the societal influence on the lived experience of SCI across and within countries. $^{48,49}$ The most appropriate way to handle such a data structure is to use extensions of standard regression models that allow for clustering of outcomes between individuals in the same cohort. For example, individual data are nested in different levels, such as the community level, country region level, and country level. One main obstacle of ordinary least squares regression analysis applied to a nested data structure to ensure that the standard errors of the regression coefficients are valid (as these will most likely be too small or too large depending on the level of the variable (within or between cluster variable) because of unobserved heterogeneity if the hierarchical data structures are ignored). Consequently, random-effects (also known as "subject-specific", "conditional", "mixed", or "multilevel") regression models will be used, which allow us to account for sources of heterogeneity that may arise at the different group and/or individual level by portioning the error term of the regression equation. ${ }^{49}$ These models are also particularly suitable for the analysis of incomplete and/or unbalanced data.

For all analyses, well-established statistical software packages such as STATA (StataCorp, Texas) and R (The R Foundation for Statistical Computing) will be used.

\section{GOVERNANCE STRUCTURE}

The InSCI governance structure aims to ensure ethical and scientific excellence, good partnership with the community of persons living with SCI, and efficient and sustainable daily and long-term study operations. The governance structure consists of six principal organizational units: a steering committee, an executive committee, a scientific committee, the study center, national study groups, and interest groups (Fig. 2). These are each described.

- Steering committee: consists of the country study leaders and representatives from individuals living with SCI, the supporting societies of ISPRM and ISCoS, the scientific committee, and the study center. It will meet twice a year. The steering committee sets the principles governing the quality and management of the overall project including the work progress of the entire LHS-SCI initiative, and oversees the results, outcomes, and dissemination of all activities performed. In addition, the steering committee approves the creation of interest groups to guide and supervise project groups within a theme of scientific content and feasibility aspects.

- Executive committee: consists of eight individuals from the steering committee who will oversee the daily operations performed by the study center. The committee meets on a regular basis, and the decision making competence lies within the framework approved by the steering committee.

- Scientific committee: consists of a chairperson, chosen national leaders, ISPRM and ISCoS representatives, and senior advisors and will be responsible for the scientific content of InSCI and all other following activities. The scientific committee will meet twice a year.

- Study center: provides operational infrastructure in administrative, statistical, and technical support. The study center reports to the executive and scientific committee.

- National study groups: are composed of the national leaders, study coordinators, delegates of the national ISCoS and ISPRM societies, patient organizations, and local persons considered beneficial for the success of the project in each country. This group unanimously decides on country-specific issues and coordinates all activities within the LHS-SCI initiative in their respective country.

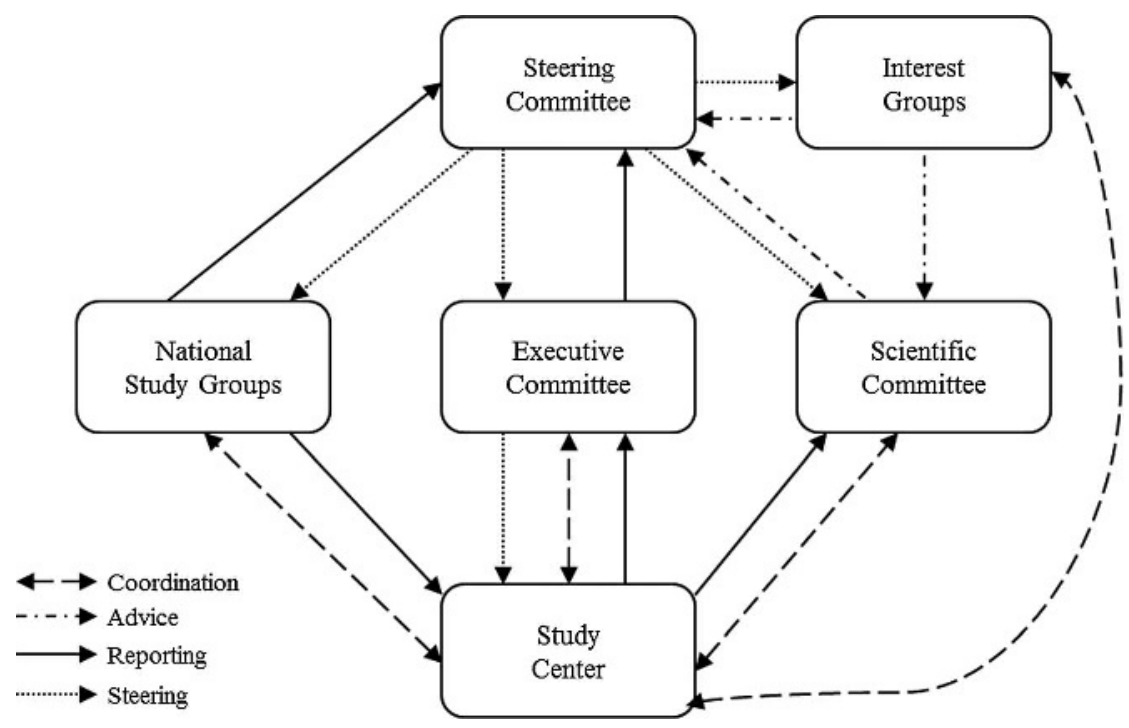

FIGURE 2. Governance structure of the LHS-SCI initiative. 
- Interest groups: provides an informal forum to develop the research agenda and funding plans as well as the implementation of nested and joint projects. Upon request, experts for a defined subject area provide scientific expertise and advice within the LHS-SCI initiative.

\section{DISCUSSION}

In this protocol paper, the overall aims and design of the InSCI community survey are detailed. InSCI will be the first standardized survey on health, functioning, and well-being of persons with SCI to be executed simultaneously in 28 countries across all six WHO world regions. As an important first step within the LHS-SCI initiative, InSCI serves as a platform to generate evidence on the lived experience of persons with SCI on an international level. To monitor goal achievement of the LHS-SCI, the InSCI community survey is foreseen to be repeated at 5 yearly intervals to evaluate progress in the health system development and service provision.

As an international survey, InSCI involves several methodological challenges that need careful consideration and planning. Each will be reviewed in turn.

\section{Representativeness of the Sample}

For cross-country comparisons, as planned for InSCI, population-based random samples would be ideal. However, as comprehensive registries on persons with SCI are lacking in many countries, such random samples are hard to obtain; and this limits the representativeness of the sampling frames. ${ }^{40}$ To guarantee feasibility and best possible adjustment to local conditions, national study groups develop country-specific strategies regarding sampling frames and sampling modes within the given InSCI guidelines. This flexibility ensures that the targeted minimal sample size of 200 eligible participants per country can be reached. This may, however, create a risk of nonrepresentative samples owing to suboptimal sampling frames. To address this issue, guidelines concerning target populations have been clearly defined, and a hierarchical suggestion of defined sampling frames have been developed. Moreover, for analysis, countries with similar sampling frames will be clustered and different scenarios and sensitivity analyses of functioning outcomes will be applied. All analyses will be adjusted for demographics and SCI characteristics, and random effects models will be used to account for unobserved heterogeneity.

\section{Addressing Nonresponse Bias}

The use of a Web-based data entry form for immediate data entry will allow the real-time monitoring of the response status of eligible subjects to ensure reminder management to nonresponders by national study groups. Each national study center develops a strategy for reminding nonresponders. As the experience of SwiSCI has shown, an elaborate reminder strategy can effectively increase participation rates. In the SwiSCI community survey, a first written reminder was followed by a telephone reminder, and this combination was an efficient way to optimize response rates. ${ }^{10}$ Based on this experience, a nonresponse rate of approximately $50 \%$ can be expected, ${ }^{8}$ and all participating countries are advised to collect survival status and basic data of eligible subjects to address and correct for unit nonresponse by inverse probability weights based on the propensity of response.

\section{Data Collection}

Whereas the InSCI questionnaire was designed to comprehensively capture the lived experience of persons with SCI considering the four guiding principles (efficiency, feasibility, comparability, truth, and discrimination) that directed the argumentative process of question selection, the validity and reliability of the InSCI questionnaire still needs to be confirmed. A first approach toward content validity of the questionnaire was established by using well-established ICF domains for SCI research purposes. Moreover, the recently completed US pilot study that assessed content validity and test-retest reliability in a sample of SCI persons as well as the country-specific pretests with SCI persons provided us with additional data on psychometric evidence and cross-cultural adaptation of the InSCI questionnaire and helped improve the final InSCI questionnaire.

Furthermore, as cross-national comparison is an aim of InSCI, data collection has to be harmonized to a certain extent so as to be comparable across countries. Thanks to the Web-based data, entry mask harmonization among the countries is possible, which may prevent unexpected bias due to different data entry layout or mechanism. The survey is planned for a common starting point in 2017 so that health systems and service provision to SCI of the participating countries can be integrated. For example, world affairs and/or significant changes in health systems affecting the countries can be integrated as covariate into the statistical modeling of outcomes. Finally, the InSCI questionnaire was developed to be culturally sensitive and understandable across countries.

\section{Comparability of Results Across Countries (Variables of the Health System and Economic Resources)}

As reported in the IPSCI report, specific data on the use of health-care services and the unmet needs of persons with SCI are often lacking and difficult to obtain, particularly in low-income countries. This results in high variability of available information. ${ }^{1}$ Yet, data about health system performance and economic resources of a country are of particular importance to understanding the societal response and may point to possible inequalities between countries. ${ }^{40}$ To determine the societal response to the lived experience of persons with SCI, variables on the health care and rehabilitation system as well as economic resource variables of the 28 participating countries have been collected and will be integrated into the statistical modeling to determine the influence of the health system on the lived experience of SCI within and between countries. ${ }^{24}$

Finally, it is important to mention that insights gained from InSCI alone will not be a complete guide to reforming practice, research, and policy in the field of disability and rehabilitation. The role of InSCI in the context of the LHS-SCI is to provide comparable evidence on the lived experience and needs of persons living with SCI. Continued research in the 
field with detailed in-depth analysis is necessary to guide further developments.

\section{CONCLUSIONS}

InSCI provides an international platform to gather evidence on the lived experience of persons with SCI and supports the LHS-SCI initiative for planning, implementing, and monitoring improvements in health care systems and service delivery. Results of the InSCI survey will provide the basis for continued research and, in particular, will support the background for stakeholder dialogs - the second pillar of LHS-SCI - that will be used to identify potential targets for interventions to improve the situation of persons living with SCI.

\section{ACKNOWLEDGMENT}

The authors thank Dr Martin $W$ G Brinkhof who strongly supported the development of the InSCI study protocol.

InSCI is part of The Learning Health System for Spinal Cord Injury, an initiative embedded in WHO's Global Disability Action Plan 2014-2021.

The members of the InSCI Steering Committee are: Christoph Gutenbrunner (ISPRM representative), Doug Brown (ISCoS representative), Gerold Stucki (Scientific committee chair), Jianan Li (co-chair, Scientific committee), Mirjam Brach (Representative Coordinating Institute, Switzerland), Christine Thyrian (Representative study center, Switzerland), Marcel W.M. Post (scientific advisor), Jerome Bickenbach (scientific advisor), Marcelo Riberto (Brazil), Juan Manuel Guzmán González (Mexico), Reuben Escorpizo (USA), James Middleton (Australia), Jianan Li (China), Eiichi Saitoh (Japan), Zee-A Han (Korea), Nazirah Binti Hasnan (Malaysia), Xiang-Hu (Shaun) Xiong (New Zealand), Luh Karunia Wahyuni (Indonesia), Apichana Kovindha (Thailand), Abderrazak Hajjioui (Morocco), Conran Joseph (South Africa), Brigitte Perrouin-Verbe (France), Christoph Gutenbrunner (Germany), Christina-Anastasia Rapidi (Greece), Iuly Treger (Israel), Mauro Zampolini (Italy), Anda Nulle (Latvia), Alvydas Juocevicius (Lithuania), Marcel Post (Netherlands), Johan K. Stanghelle (Norway), Piotr Tederko (Poland), Jorge Lains (Portugal), Mercè Avellanet (Spain), Per Ertzgaard (Sweden), and Michael Baumberger (Switzerland).

\section{REFERENCES}

1. Bickenbach JE, Officer A, Shakespears T, et al: International Perspectives on Spinal Cord Injury, Geneva, WHO Press, 2013

2. Chamberlain JD, Meier S, Mader L, et al: Mortality and longevity after a spinal cord injury: systematic review and meta-analysis. Neuroepidemiology 2015;44:182-98

3. Barclay L, McDonald R, Lentin P: Social and community participation following spinal cord injury: a critical review. Int J Rehabil Res 2015;38:1-19

4. Marti A, Reinhardt JD, Graf S, et al: To work or not to work: labour market participation of people with spinal cord injury living in Switzerland. Spinal Cord 2012;50:521-6

5. World Health Organization: World Report on Disability. Geneva, WHO, 2011

6. Institute of Medicine: Best Care at Lower Cost. Washington, DC, National Academies Press, 2012

7. Prodinger B, Cieza A, Oberhauser C, et al: Toward the International Classification of Functioning, Disability and Health (ICF) Rehabilitation Set: a minimal generic set of domains for rehabilitation as a health strategy. Arch Phys Med Rehabil 2016;97:875-84

8. Brinkhof MW, Fekete C, Chamberlain JD, et al: Swiss national community survey on functioning after spinal cord injury: protocol, characteristics of participants and determinants of nonresponse. J Rehabil Med 2016;48:120-30

9. Post MW, Brinkhof MW, von Elm E, et al: Design of the Swiss Spinal Cord Injury Cohort Study. Am J Phys Med Rehabil 2011;90(suppl):S5-16
10. Fekete C, Segerer W, Gemperli A, et al: Participation rates, response bias and response behaviours in the community survey of the Swiss Spinal Cord Injury Cohort Study (SwiSCI) BMC Med Res Methodol 2015;15:80

11. Prodinger B, Ballert CS, Brach M, et al: Toward standardized reporting of a cohort study on functioning: the Swiss Spinal Cord Injury Cohort Study (SwiSCI) as a case in point. $J$ Rehabil Med 2016;48:189-96

12. WHO: ICF - International Classification of Functioning, Disability and Health. Geneva, WHO Press, 2001

13. Geyh S, Peter C, Muller R, et al: The personal factors of the International Classification of Functioning, Disability and Health in the literature - a systematic review and content analysis. Disabil Rehabil 2011;33:1089-102

14. Noonan VK, Thorogood NP, Fingas M, et al: The validity of administrative data to classify patients with spinal column and cord injuries. J Neurotrauma 2013;30:173-80

15. Hagen EM, Rekand T, Gilhus NE, et al: Diagnostic coding accuracy for traumatic spinal cord injuries. Spinal Cord 2009;47:367-71

16. Noreau L, Noonan VK, Cobb J, et al: Spinal Cord Injury Community Survey: a national, comprehensive study to portray the lives of Canadians with spinal cord injury. Top Spinal Cord Inj Rehabil 2014;20:249-64

17. World Medical Association (WMA): WMA Declaration of Helsinki-Ethical Principles for Medical Research Involving Human Subjects. (Adopted by the 18th WMA General Assembly, Helsinki, Finland, June 1964 and amended by the: 29th WMA General Assembly, Tokyo, Japan, October 1975; 35th WMA General Assembly, Venice, Italy, October 1983; 41st WMA General Assembly, Hong Kong, September 1989; 48th WMA General Assembly, Somerset West, Republic of South Africa, October 1996; 52nd WMA General Assembly, Edinburgh, Scotland, October 2000; 53rd WMA General Assembly, Washington DC, October 2002 (Note of Clarification added); 55th WMA General Assembly, Tokyo, Japan, October 2004 (Note of Clarification added); 59th WMA General Assembly, Seoul, Republic of Korea, October 2008; 64th WMA General Assembly, Fortaleza, Brazil, October 2013). 1964

18. World Health Organization: WHO World Health Survey. 2016; Available at: http://www.who. int/healthinfo/survey/en/. Accessed June 17, 2016

19. Cieza A, Kirchberger I, Biering-Sørensen F, et al: ICF Core Sets for individuals with spinal cord injury in the long-term context. Spinal Cord 2010;48:305-12

20. Geyh S, Müller R, Peter C, et al: Capturing the psychologic-personal perspective in spinal cord injury. Am J Phys Med Rehabil 2011;90(Suppl):S79-96

21. Edwards PJ, Roberts I, Clarke MJ, et al: Methods to increase response to postal and electronic questionnaires. Cochrane Database Syst Rev 2009:MR000008

22. Jepson C, Asch DA, Hershey JC, et al: In a mailed physician survey, questionnaire length had a threshold effect on response rate. J Clin Epidemiol 2005;58:103-5

23. Fekete C, Boldt C, Post M, et al: How to measure what matters: development and application of guiding principles to select measurement instruments in an epidemiologic study on functioning. Am J Phys Med Rehabil 2011;90(suppl):S29-38

24. Fekete C, Post MWM, Bickenbach J, et al: A structured approach to capture the lived experience of spinal cord injury: data model and questionnaire of the International Spinal Cord Injury community survey. Am J Phys Med Rehabil 2017;96(Suppl):S5-16

25. Ware JE Jr, Sherbourne CD: The MOS 36-item short-form health survey (SF-36). I Conceptual framework and item selection. Med Care 1992;30:473-83

26. Cleeland CS, Ryan KM: Pain assessment: global use of the Brief Pain Inventory. Ann Acad Med Singapore 1994;23:129-38

27. Kalpakjian CZ, Scelza WM, Forchheimer MB, et al: Preliminary reliability and validity of a Spinal Cord Injury Secondary Conditions Scale. J Spinal Cord Med 2007;30:131-9

28. World Health Organization, The World Bank: Model Disability Survey (MDS). Available at: http://www.who.int/disabilities/data/mds/en/. Accessed March 24, 2016

29. Fekete C, Eriks-Hoogland I, Baumberger M, et al: Development and validation of a self-report version of the Spinal Cord Independence Measure (SCIM III). Spinal Cord 2013;51:40-7

30. Jette AM, Slavin MD, Ni P, et al: Development and initial evaluation of the SCI-FI/AT. J Spinal Cord Med 2015;38:409-18

31. Ballert CS, Post MW, Brinkhof MW, et al: Psychometric properties of the Nottwil Environmental Factors Inventory Short Form. Arch Phys Med Rehabil 2015;96:233-40

32. Schwarzer R, Jerusalem M: Generalized Self-Efficacy scale. in, Weinman J, Wright S, Johnston M, eds. Measures in Health Psychology: A User's Portfolio. Causal and Control Beliefs. Windsor, UK, NFER-NELSON, 1995:35-7

33. Middleton J, Tate R, Geraghty T: Self-efficacy and spinal cord injury: psychometric properties of a new scale. Rehabil Psychol Health Med 2003;48:281-8

34. Malone GP, Pillow D, Osman A: The General Belongingness Scale (GBS): assessing achieved belongingness. Personal Individ Differ 2012;52:311-6

35. World Health Organization: The World Health Organization Quality of Life (WHOQoL)-BREF. Geneva, WHO, 2004

36. Epstein J, Osborne RH, Elsworth GR, et al: Cross-cultural adaptation of the Health Education Impact Questionnaire: experimental study showed expert committee, not back-translation, added value. J Clin Epidemiol 2015;68:360-9

37. Pacheco Barzallo D, Gross-Hemmi MH: The cross-cultural societal response to SCI: health and related systems. Am J Phys Med Rehabil 2017;96(Suppl):S41-54

38. Shah AD, Bartlett JW, Carpenter J, et al: Comparison of random forest and parametric imputation models for imputing missing data using MICE: A CALIBER Study. Am J Epidemiol 2014;179:764-74

39. Stekhoven DJ, Bühlmann P: MissForest-non-parametric missing value imputation fo mixed-type data. Bioinformatics 2012;28:112-8 
40. Reinhardt JD, Mansmann U, Fellinghauer BA, et al: Functioning and disability in people living with spinal cord injury in high- and low-resourced countries: a comparative analysis of 14 countries. Int J Public Health 2011;56:341-52

41. Fellinghauer BA, Reinhardt JD, Stucki G: Towards an epidemiology of functioning, in Franchignoni F, (ed): Advances in Rehabilitation. Research Issues in Physical \& Rehabilitation Medicine. 2010:53-67

42. DeVivo M, Biering-Sørensen F, Charlifue S, et al: International Spinal Cord Injury core data set. Spinal Cord 2016;44:535-40

43. Kalisch M, Mächler M, Colombo D, et al: Causal inference using graphical models with the R package pcalg. Journal of Statistical Software 2012;47

44. Maathuis MH, Kalisch M, Bühlmann P: Estimating high-dimensional intervention effects from observational data. Ann Statist 2009;37:3133-64
45. Prodinger B, Ballert CS, Brach M, et al: Toward standardized reporting for a cohort study on functioning: the Swiss Spinal Cord Injury Cohort Study. J Rehabil Med 2016;48:189-96

46. Christensen KB, Kreiner S, Meshab M: Rasch Models in Health, London, ISTE Ltd and John Wiley \& Sons Inc, 2013

47. Peter C, Schulenberg SE, Buchanan EM, et al: Rasch analysis of measurement instruments capturing psychological personal factors in persons with spinal cord injury. $J$ Rehabil Med 2016;48:175-88

48. Kirkwood BR, Sterne JAC: Essential Medical Statistics, Malden, MA, Blackwel Science, 2003

49. Gelman A, Hill J: Data Analysis Using Regression and Multilevel/Hierarchical Models, Cambridge, UK, Cambridge University Press, 2007 